\title{
COVID-19 and Dentistry
}

An article commissioned by the Journal of the College of General Practitioners of Sir Lanka

In December 2019, patients in Wuhan, Hubei Province, Central China exhibited symptoms of viral pneumonia similar to Middle East Respiratory Syndrome Coronavirus (MERS-CoV). On 1st January 2020, the seafood market was closed for decontamination. The Chinese Centre for Disease Control and Prevention announced they had identified a novel coronavirus on 9th January 2020.

Researchers undertook genomic sequencing and determined the causative pathogen was a virus which was related to the coronavirus family (the same responsible for MERS and SARS). Hence, it was named novel coronavirus 2019 (2019 n-CoV). 1

It was subsequently renamed, severe acute respiratory syndrome coronavirus 2 (SARS-CoV-2), the virus responsible for Coronavirus Disease 2019 (COVID-19). On 23rd January 2020, 655 cases and 18 deaths were reported in China and 9 other countries. 2 A mathematical model determined that it was ineffective to isolate

confirmed positive patients and trace their contacts alone to prevent new outbreaks. The delays from symptom onset to isolation were the predominant reasons cited. Therefore, lockdowns and local restrictions were necessary to control this pandemic. 3 On 11th march 2020, the World Health Organisation declared SARS-CoV-2 a pandemic. As of 4th March 2021, the WHO have reported 114, 853, 685 confirmed SARS-CoV-2 cases including 2, 554, 694 deaths globally. 4

SARS-CoV-2 is transmitted through direct contact by respiratory droplets (when a patient coughs, sneezes or talks) which typically cannot traverse more than two metres. Indirect transmission occurs through SARS-CoV-2 remaining viable in droplets and being suspended in the air and landing on surfaces. If a person touches a surface contaminated with SARS-CoV-2 and then touches a mucous membrane (e.g. eyes, nose or mouth) then they are at high risk of acquiring SARS-CoV-2.5

In a closed space environment, the virus (aerosol) can remain in the air as clusters for a considerable time. 6 Based on these identified modes of transmission, dentistry is a high-risk profession given the close proximity of dentists to the patient (less than two metres), direct inspection of the oral cavity and oropharynx and potential for dental procedures to stimulate the cough reflex. Furthermore, the use of dental instruments such as handpieces and ultrasonic scalers creates an aerosol which can scatter the virus into the air. There is also a high volume of patients seen in a dental clinic, small space and lack of ventilation compounding the risk of viral transmission.

There are measurable steps dental practices can take to mitigate the risk of SARS CoV-2 transmission in their dental practice.7, 8 Firstly, all staff should have a daily COVID-19 questionnaire for risk assessment, non-touch forehead temperature taken and ideally, a rapid SARS-CoV-2 test which should be recorded

\footnotetext{
Dr Manas Dave NIHR Academic Clinical Fellow in Oral and Maxillofacial Pathology, The University of Manchester, United Kingdom.

Dr Senathirajah Ariyaratnam - BDS, MDS, FDSRCS, SFHEA, NTF. Clinical Senior

Lecturer/Specialist in Oral Medicine, The University of Manchester/Manchester Foundation Trust, United Kingdom.
} 
in the dental clinics risk assessment for COVID-19 policy folder. Staffs who have any signs they may be positive for COVID-19 need to be sent home immediately (ideally by private car and not public transport). The local (country) quarantine guidelines and reporting policy should be followed. Under no circumstances should any staff or patient who shows signs of COVID-19 infection be allowed to enter the dental clinic.

For patients, tele- dentistry and triage protocols should be implemented. This includes a telephone screen to assess patients for symptoms consistent with COVID-19 and also their medical history check to assess their vulnerability. If patients inform of symptoms that are suggestive of COVID-19, they should have delayed dental care where possible to prevent spreading the infection. Dentists may wish to see vulnerable patients (patients with comorbidity) at a separate time to the rest of their patient cohort and preferably avoiding times when AGPs are being conducted to reduce their risk of viral infection.

It is important to recognise that high percentage of patients can be asymptomatic carriers hence the telephone triage is only a partial mitigation strategy. Patients should be advised to limit the number of visitors they bring and to be told hand sanitation and the use of a facemask is compulsory. Extra luggage should not be brought in the dental clinic. On arrival, non-touch forehead temperature should be checked along with symptoms through a questionnaire. Any patients demonstrating new symptoms consistent with COVID-19 advised to leave the practice and enter isolation or if an emergency, visit a facility that has provisions to provide the adequate cross-infection care (such as a hospital setting).

Dental practices should supply at least $60 \%$ concentration alcohol hand gel and instruct all patients to use this on arrival at the surgery. A protective physical barrier such as a glass shield should be placed to cover reception areas and limit close contact between patients and staff where reasonably practicable.

The waiting room chairs should be placed two metres apart and patients should be instructed to wait outside the surgery, only attending at their specified appointment time to prevent lots of patient gathering in the waiting room. There should be adequate ventilation (either natural or mechanical ventilation) available and all objects (such as magazines, children's toys etc.) should be removed. If dental clinics have the facility to undertake rapid SARS-CoV-2 testing (lateral flow -antigen test), this should be undertaken as an adjunct to all other precautions to identify patients who may be asymptomatic carriers.

Dentists should avoid aerosol generating procedures (AGPs) and minimise aerosol exposure whenever possible. These include:

* High speed dental handpieces

* Air/water syringe

* Ultrasonic scalers

AGP specific slots should be booked at the end of the day to allow a long fallow time and appropriate clinical workflow. These are discussed in detail later. There are proactive measures dentists can take to mitigate the risk of SARS-CoV-2 transmission during AGPs.

These include:

* Using rubber dam

* High volume suction (orifice diameter $8 \mathrm{~mm}$ )

* Hand instrument use only (e.g. atraumatic restorative technique)

* Not undertaking AGPs in rooms without windows unless additional calibrated quality ventilation is 
Present

There is no evidence so far to suggest that the use of pre-procedural mouth rinses helps reduce the risk of SARS-CoV-2 transmission. Additionally, dental radiography can still be undertaken as required. High dose extra oral radiography should not be substituted for intra oral radiography as the dental clinician should have appropriate PPE in place to mitigate for such risk. The additional radiation dosage would not be justified. ${ }^{9}$

Regarding personal protective equipment, dental professionals (including dentists, nurses, hygienists, therapists and all other specialities with a clinical role) should wear standard personal protective equipment (PPE) for low risk procedures such as examinations and non-aerosol generating procedures. This includes a disposable quality facemask, gloves, safety eyewear and appropriate clinical attire (that is only used in the dental clinic and not taken outside). For higher risk procedures (such as when undertaking an AGP), FFP2/FFP3/PAPR visor should be worn along with a gown, gloves, safety eye wear and separate clinical attire.

After treatment, standard decontamination protocols should be followed. Additionally, the dentist should discard their gowns and other PPE in the surgery however keep their facemask on until they have left the dental treatment room to prevent inhalation of SARS-CoV-2 which has been suspended in the air. All staff should leave the clinical room and this should be closed to allow for a fallow time. This is the time required for any SARS-CoV-2 to settle on surfaces from the air. The fallow time is calculated depending on the procedure and the duration of the time it was conducted, ventilation of the room and the overall level of mitigation employed. It is important that dental clinics are kept clear of all non-viable contents. This includes paper records which can harbour SARS-CoV-2. At the end of each clinical day, dental professionals should wash their clinical attire at the highest possible temperature and not wear this outside of the dental clinic.

The following guidance is provided by the Faculty of General Dental Practice (UK). ${ }^{8}$

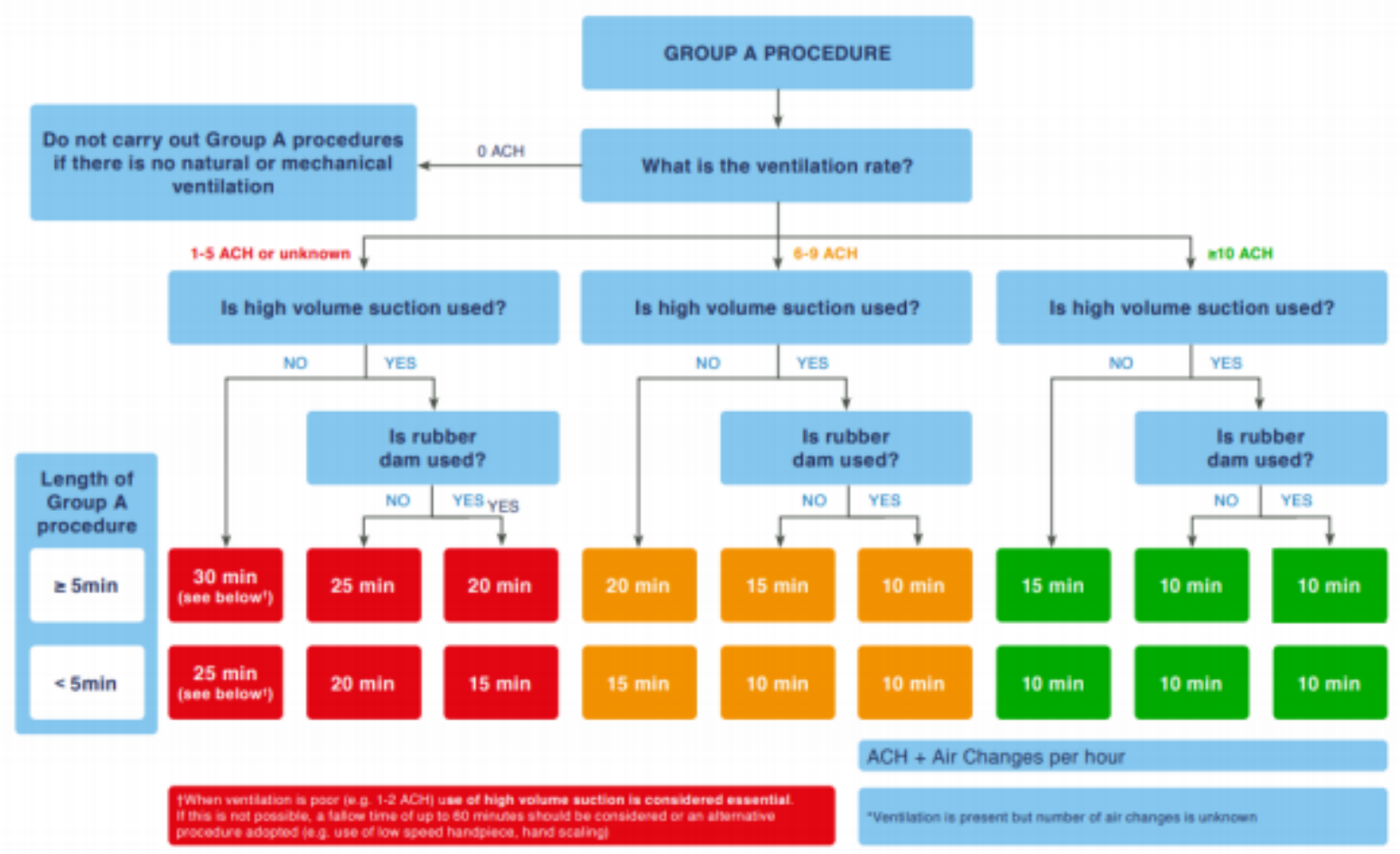

Figure 1: SDCEP, Mitigation of Aerosol Generating Procedures in Dentistry, A Rapid Review, Version 1.0. 25/9/20 


\begin{tabular}{|c|c|c|}
\hline PAOCEDURE & LOWER RISK (aerosol exposure) & 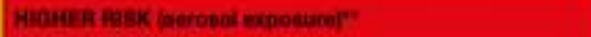 \\
\hline Oal hypiene instruesios & 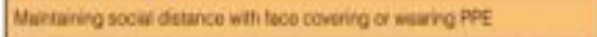 & $x$ \\
\hline Toeth Prophylaces & 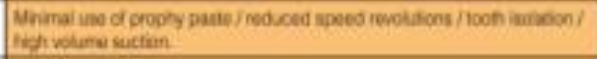 & 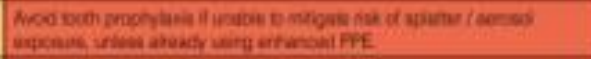 \\
\hline Eutra-ara radiography / CnCt & 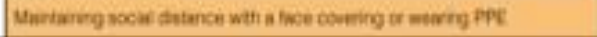 & $x$ \\
\hline $\begin{array}{l}\text { hiba-ord adiography } \\
\text { Fink ascoss the reed in relotion to COMD-19) }\end{array}$ & 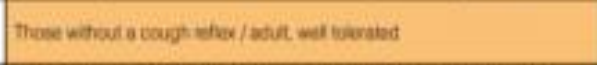 & 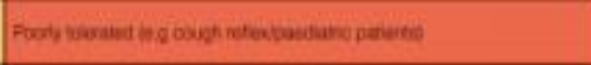 \\
\hline Duntal pholography & 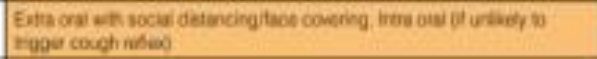 & 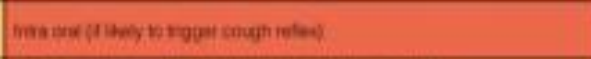 \\
\hline Cinical enamination & 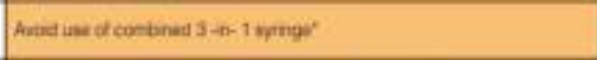 & 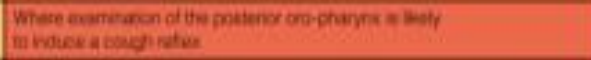 \\
\hline Dinet restoration of a tooth & 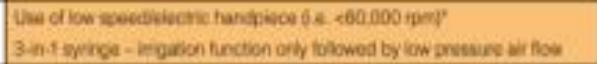 & 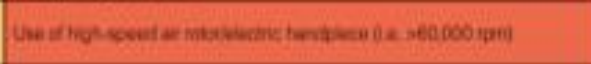 \\
\hline APl cementafon crom or bridps & 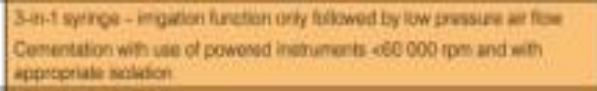 & 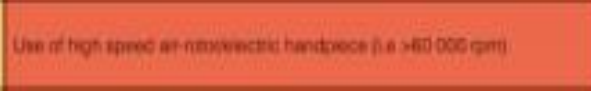 \\
\hline Resovable prosthodortics & Wien well Benatod br al atages & Whes poorly bienaded for at lasas \\
\hline Adfuntment and mpar of monovatie pruethesis & 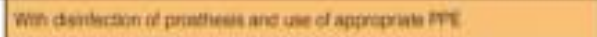 & $x$ \\
\hline Dutrastion of tooth & 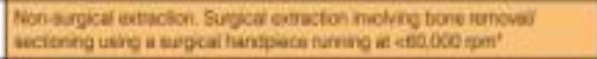 & 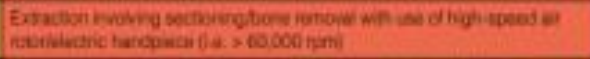 \\
\hline Reatoration or repair of inplart reained prostives & 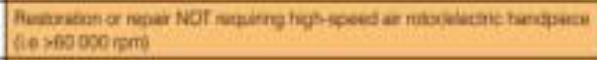 & 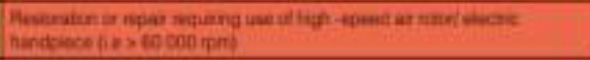 \\
\hline Inpint placement & 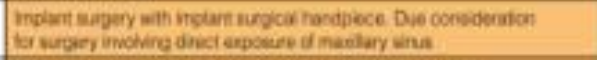 & nese arigeryus: \\
\hline Endodonte procedurn & 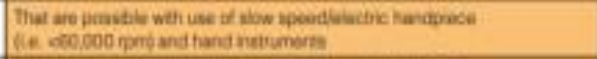 & 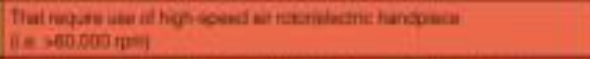 \\
\hline Periodomal procedures & 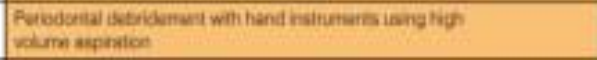 & 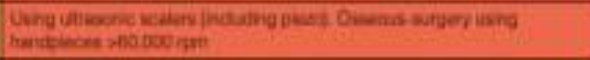 \\
\hline Fasume sealarts & 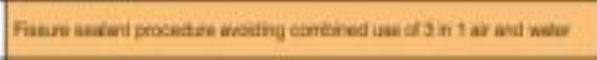 & 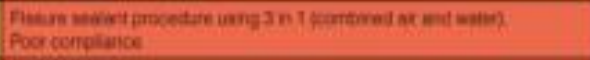 \\
\hline Mrimaly insanive matration & 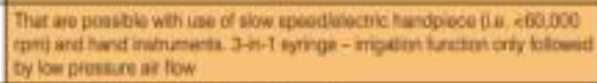 & 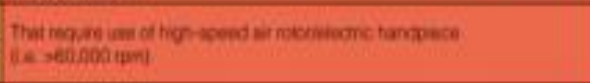 \\
\hline heive and drain abovess & 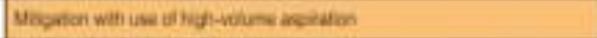 & $x$ \\
\hline Oetrodsntic heatment & 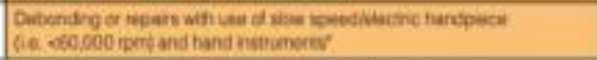 & 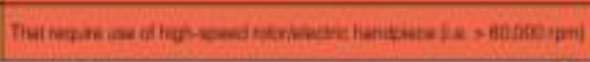 \\
\hline
\end{tabular}

Figure 2: Risk stratification for Aerosol Generation Exposure (AGEs)

\section{Vaccination protocol}

It is of note that the vaccination is not recommended (as of the date of 5th March 2021) for children (16 or 18 years of age depending on the vaccine) and those with a history of severe allergic reaction to any component of the vaccine. For pregnant women, there is limited data available and their personal vulnerability to COVID-19 needs to be taken into consideration for a personalised decision in discussion with their healthcare provider.10

The Pfizer-BioNTech vaccine demonstrated 95\% efficacy against COVID-19. Israel's vaccination programme showed that two doses of the Pfizer-BioNTech vaccine reduced symptomatic cases by $94 \%$, hospital admissions by $87 \%$ and severe COVID-19 by $92 \% .11$ A full summary of the PfizerBioNTech vaccine is provided: https://www.linkedin.com/pulse/publication-summarysafetyefficacy-bnt162b2-mrna covid- 19 - manas- dave/ 
For the Oxford-AstraZeneca vaccine, a single standard dose of the vaccine provided $76 \%$ protection against symptomatic COVID-19 in the first 90 days. Significant degree of protection will appear only after 3 weeks following vaccination. The vaccine efficacy reached $82.4 \%$ after the second dose. 12

It is recommended that dental clinicians take their role as healthcare providers in society to proactively remove misinformation about the COVID-19 vaccination programme. Wherever possible, the dental team need to provide personalised information and advice to empower patients and the local population. Posters should be displayed in the waiting room to help reinforce the importance of infection prevention, accessing health care facilities if suspected COVID-19 infection and accessing vaccination programmes.

\section{Domestic violence}

There have been numerous reports of COVID-19 lockdown resulting in an increase in domestic violence cases. Dental clinicians need to be vigilant about signs and symptoms of non-accidental injury and have an appropriate safeguarding policy in place to escalate matters as appropriate. This includes both men and women and children. 13

\section{References}

1.Zhou P, Yang XL, Wang XG, Hu B, Zhang L, Zhang W, et al. A pneumonia outbreak associated with a new coronavirus of probable bat origin. Nature. 2020.

2.Gralinski LE, Menachery VD. Return of the Coronavirus: 2019-nCoV. Viruses. 2020;12(2):135.

3. Hellewell J, Abbott S, Gimma A, Bosse NI, Jarvis CI, Russell TW, et al. Feasibility of controlling COVID-19 outbreaks by isolation of cases and contacts. The Lancet Global Health. 2020;8(4):e488-e96.

4. World Health Organisation. WHO Coronavirus Disease (COVID-19) Dashboard [Online]. 2020 [cited 20205 March]. Available from: https://covidl9.who.int/.

5.Lotfi M, Hamblin MR, Rezaei N. COVID-19: Transmission, prevention, and potential therapeutic opportunities. Clin Chim Acta. 2020;508:254-66.

6. The Lancet Respiratory M. COVID-19 transmission-up in the air. The Lancet Respiratory medicine. 2020;8(12):1159.

7.Centers for Disease Control and Prevention. Guidance for Dental Settings 2020 [cited 20214 March]. Available from: https://www. cdc.gov/coronavirus/2019 ncov/hcp/dental-settings.html.

8.FGDP(UK). Implications of COVID-19 for the safe management of general dental practice - a practical guide 2020 [cited 20214 March]. Available from: https://www.fgdp.org.uk/implications-covid-19-safe-management-general-dental practice-practical-guide.

9.Dave M, Coulthard P, Patel N, Seoudi N, Horner K. Letter to the Editor: Use of Dental Radiography in the COVID-19 Pandemic. Journal of Dental Research. 2020:0022034520923323.

10. World Health Organisation. he Oxford/AstraZeneca COVID-19 vaccine: what you need to know 2021 [cited 20204 March]. Available from: https://www.who.int/news-room/feature-stories/detail/the-oxford-astrazeneca-covid19-vaccine-what-you-need-toknow.

11. Mahase E. Covid-19: Where are we on vaccines and variants? BMJ (Clinical research ed). 2021;372:n597.

12. Wise J. Covid-19: New data on Oxford AstraZeneca vaccine backs 12 week dosing interval. BMJ (Clinical research ed). 2021;372:n326.

13. Dave M, Patel N. Domestic violence during the covid-19 pandemic United Kingdom: BMJ 2020 [cited 2020 May 14]. Available from: https://blogs.bmj.com/bmj/2020/05/07/domestic-violence-during-the-covid-19 pandemic/. 